\title{
Analyses of ERG in a patient with intraocular lymphoma
}

This article was published in the following Dove Press journal:

Clinical Ophthalmology

6 April 2010

Number of times this article has been viewed

\section{Chieko Yasuda \\ Shinji Ueno \\ Mineo Kondo \\ Nagako Kondo \\ Chang-Hua Piao \\ Hiroko Terasaki \\ Department of Ophthalmology, Nagoya University School of Medicine, Nagoya, Japan}

Correspondence: Shinji Ueno, Department of Ophthalmology, Nagoya University Graduate School of Medicine, 65 Tsuruma-cho, Showa-ku,

Nagoya 466-8550, Japan

$\mathrm{Tel}+8 \mathrm{I}-52-744-227 \mathrm{I}$

Fax +8I-52-744-2278

Email ueno@med.nagoya-u.ac.jp
Purpose: To follow the changes in the electroretinograms (ERGs) in a patient with primary intraocular lymphoma (PIOL) who had a complete remission after chemotherapy.

Methods: ERGs were recorded in a 41-year-old woman with PIOL during and after complete remission with chemotherapy. The patient was diagnosed with PIOL from both the ocular signs and the medical history of cranial lymphoma.

Results: The ERGs were depressed in the subject. The amplitudes of the bright white flash b-waves were smaller than the a-waves, resulting in a "negative type" ERG. Six weeks after the beginning of chemotherapy, the ocular changes had resolved, and the ERGs, although not of the "negative type", still showed signs that the amplitude had not returned to normal levels.

Conclusion: The negative type ERGs indicated that the inner retina had been damaged to a greater extent than the outer retina. In the convalescent stage, when the ocular manifestations were resolved, the ERGs were still not fully recovered. Although only one case was studied, we suggest that ERGs can be used to evaluate and follow patients with a PIOL.

Keywords: electroretinogram, primary intraocular lymphoma, uveitis masquerade syndrome

\section{Introduction}

A primary intraocular lymphoma (PIOL) is a subtype of primary non-Hodgkin's lymphomas of the central nervous system in which the retina, vitreous, and/or optic nerve head may be involved. ${ }^{1-3}$ Patients with a PIOL typically present with a form of uveitis and vitritis that is unresponsive to corticosteroid therapy, thus the so-called uveitis masquerade syndrome. This can occur together with, or independently of, a primary cerebral nervous system lymphoma. The disease has a poor prognosis, and patients normally die as a result the growth of the cerebral lymphoma with a mean survival of 20 months from the diagnosis of the ocular disorder. ${ }^{4}$

As best we know, electroretinography (ERG) has not been used to follow the changes in retinal function during the course of PIOL. We present the changes in the ERGs during and after a complete remission of PIOL with chemotherapy.

\section{Case report}

The patient, a 41-year-old woman, had been diagnosed with a primary intracranial malignant lymphoma (non-Hodgkins, diffuse medium-sized B cell lymphoma) for three years. She presented with a 3-week history of blurred vision and photophobia, primarily in her right eye. Her best-corrected visual acuities were 20/60 OD and 20/20 OS. The intraocular pressure was normal in both eyes. Slit-lamp examination showed mild vitritis in the right eye, and ophthalmoscopy showed no abnormalities. 
After 3 weeks of observation the right fundus had developed whitish exudates, massive subretinal infiltration from a macular lesion, and slight swelling of the optic disc. Cells were detected in the anterior chamber, but the fundus of the left eye was normal (Figure 1). The Goldmann visual field of the right eye had a dense central relative scotoma, but was almost normal in the left eye (Figure 2).

Full-field ERGs were recorded according to International Society for Clinical Electrophysiology of Vision (ISCEV) standards. ${ }^{5}$ The scotopic, bright white, cone, and $30 \mathrm{~Hz}$ flicker ERGs 3 weeks after the initial visit are shown in Figure 3. The amplitudes of the different ERG components were reduced in both eyes, but to a greater extent in the right eye. The amplitude of the bright white flash b-waves was distinctly reduced, and was smaller than the a-waves, resulting in the "negative type" ERG in both eyes. This indicated that the functional depression was greater in the inner retina than the outer retina. ${ }^{6}$

Focal macular ERGs were recorded to assess macular function as described in Miyake et al. ${ }^{7}$ The ERG from the right eye was almost nonrecordable, and that of left eye was about one-half the normal amplitude (Figure 3; Focal macular ERGs).

Because of the rapid decrease in the visual acuity of the right eye to $20 / 600$, and the increasing retinal infiltration and exudations since the previous examination, diagnostic vitrectomy was performed (Figure 4). Cytological examination of the vitreal specimen did not show any abnormal lymphocytes or monocytes, in addition to which flow cytometry did not show monoclonality. The cytokine analysis was negative without a specific increase in the IL10/IL6 ratio. Although the results of the cytological examinations were negative, a primary intraocular lymphoma was strongly suspected.

Eight weeks after the first visit, chemotherapy with cyclophosphamide, cytosine arabinoside, etoposide, and dexamethasone was started. Four days after the treatment, the visual symptoms and ophthalmoscopic appearance of the fundus improved. Six weeks after beginning chemotherapy, her best-corrected visual acuity improved to 20/20 in both eyes. The ocular lesions were completely resolved, leaving atrophic pigmentary changes (Figure 5). A relative central scotoma still remained in the right eye, but the visual field
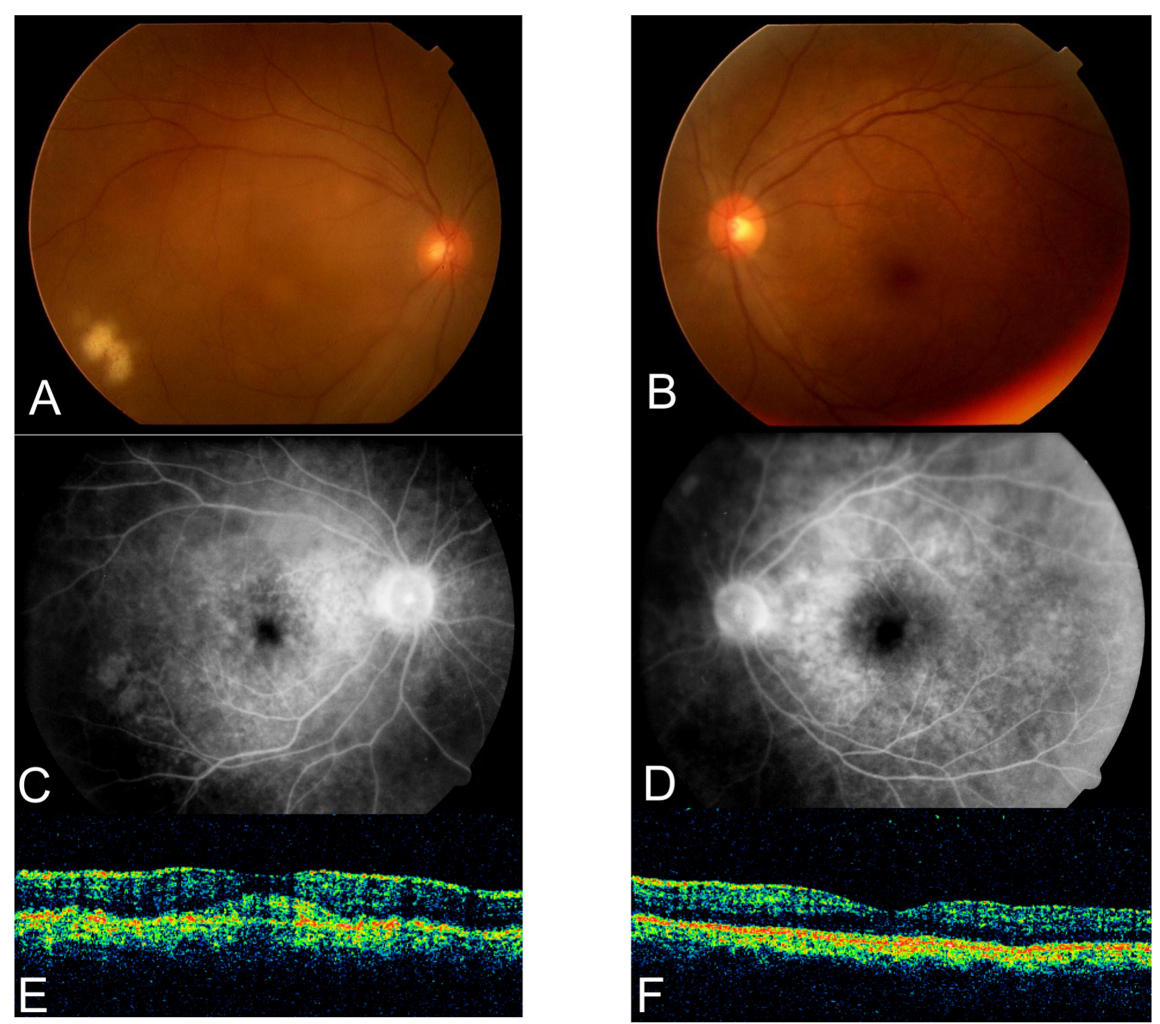

Figure I Fundus photograph of the right A) and the left eyes B). The right fundus shows whitish exudates, massive subretinal infiltration under the macular lesion, and slight disc swelling. The left fundus is normal. Fluorescein angiography of the right $\mathbf{C}$ ) and left eyes $\mathbf{D})$ Slight leakage of fluorescein can be seen on the right optic disc. The left eye is normal. Optical coherence tomography of the macular area of the right $\mathbf{E}$ ) and left eye $\mathbf{F}$ ). Massive subretinal infiltration is present in the right eye. The left eye is normal. 


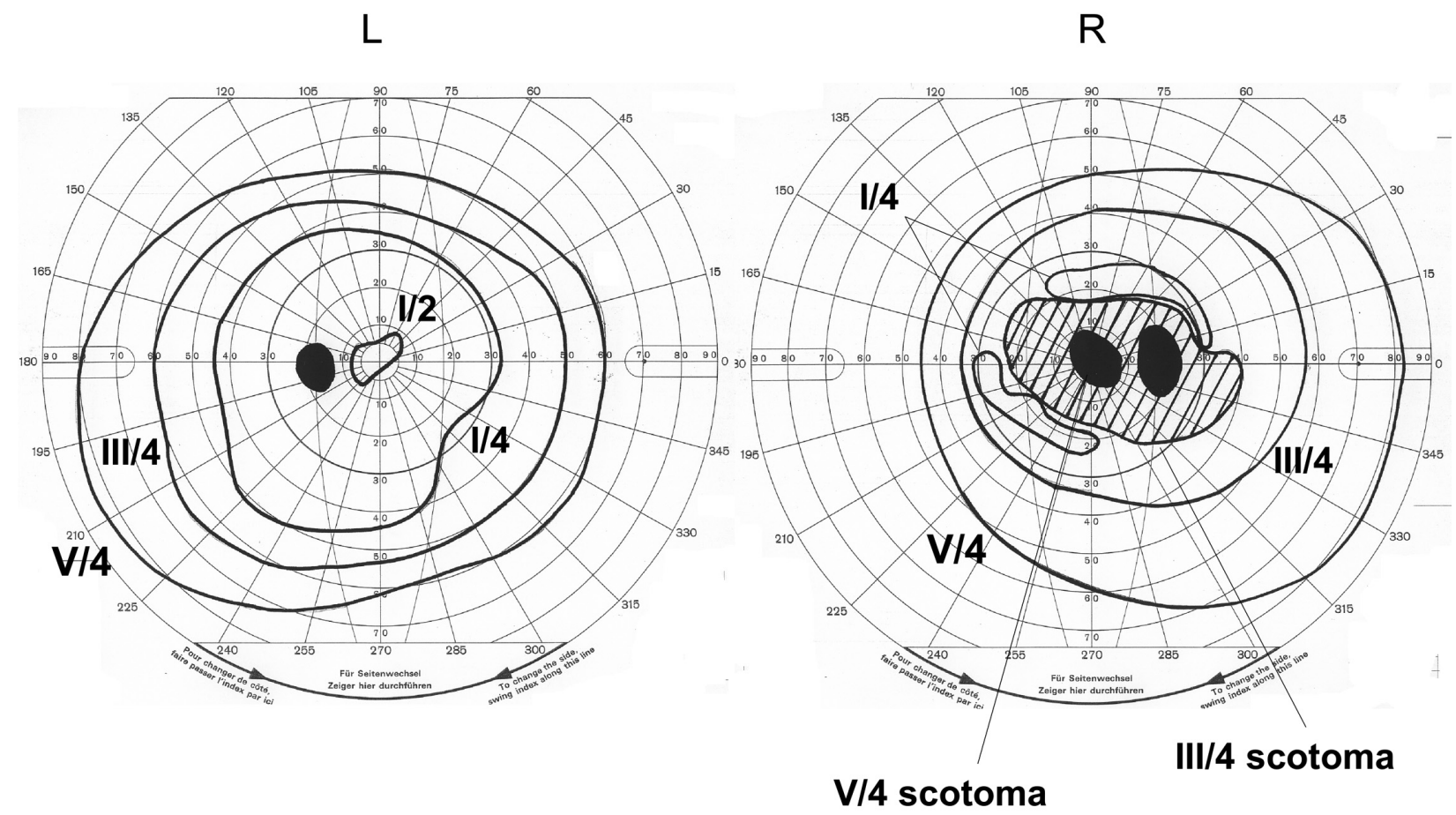

Figure 2 Goldmann perimetry showing a central scotoma in the right eye. The visual field of left eye is normal.

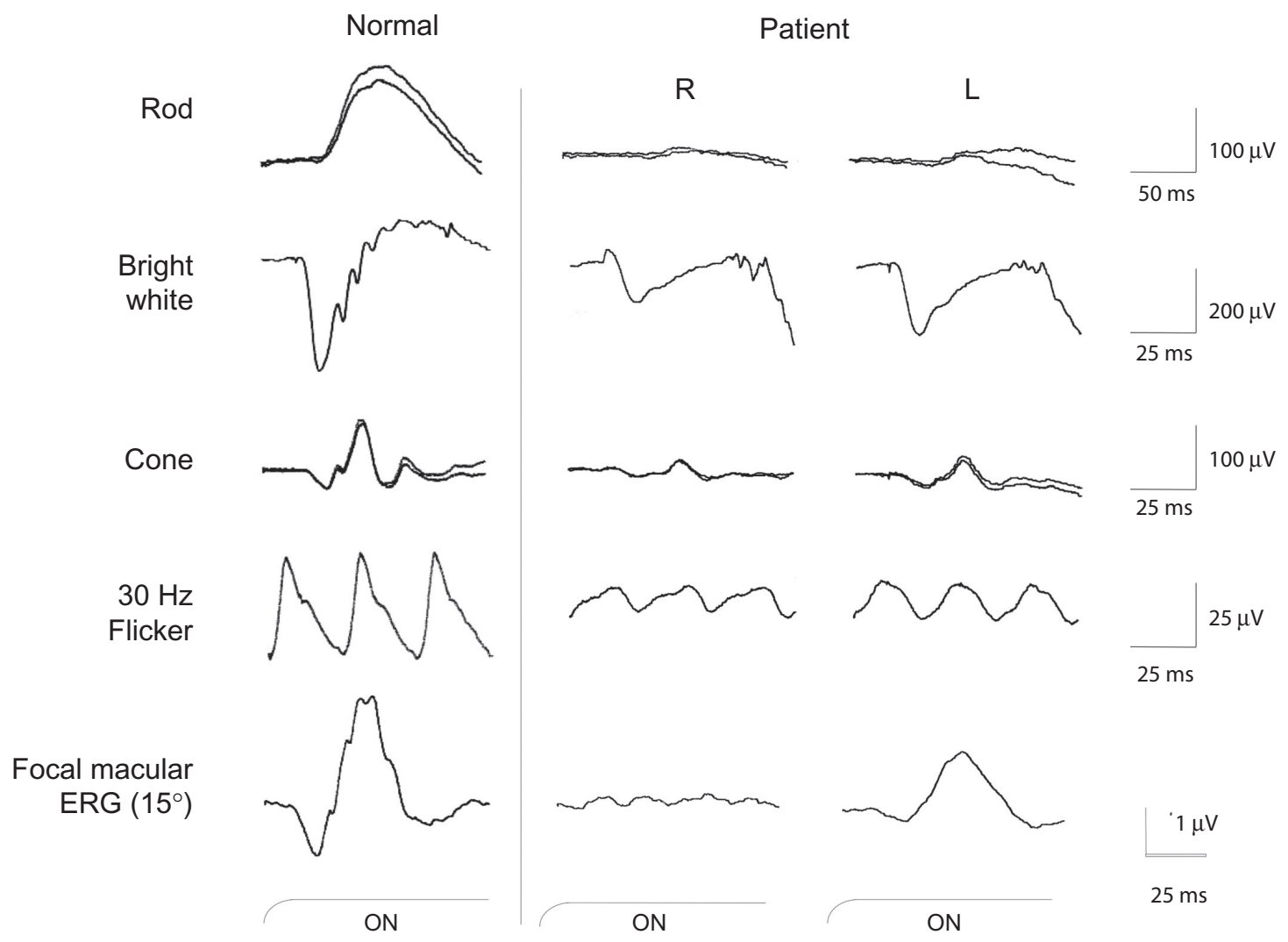

Figure 3 Full-field ERGs (rod, bright white, cone and $30 \mathrm{~Hz}$ flicker) recorded according to International Society for Clinical Electrophysiology of Vision (ISCEV) standards. Compared to the normal ERGs, the amplitudes of all components were reduced especially in the right eye. Focal macular ERGs elicited by a I5 degree stimulus spot were recorded from both eyes (bottom row). A marked reduction of amplitude can be seen in the right eye. 

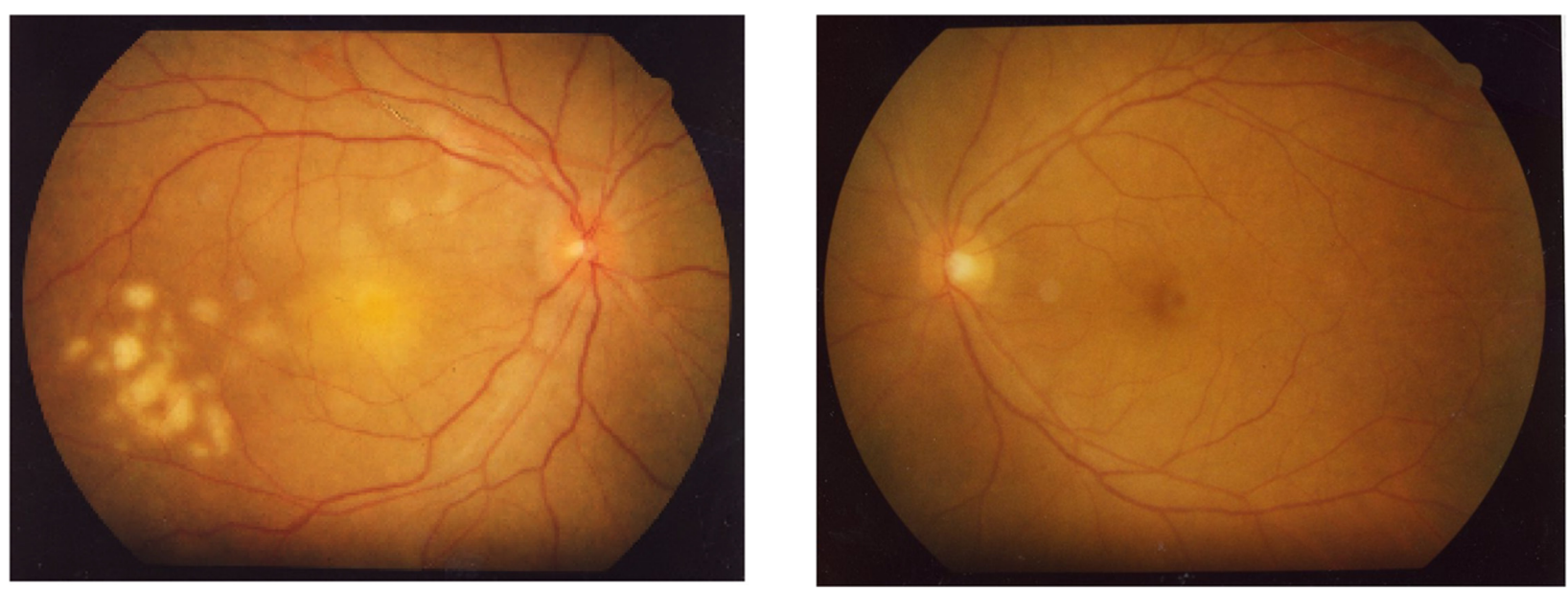

Figure 4 Fundus photograph after vitrectomy of the right eye. The retinal infiltrates and exudates have progressed in the right eye within one week since the previous examination. The left eye fundus had no abnormality.

sensitivity had improved. The cells in the anterior chamber of the left eye had also disappeared.

Although the rod and cone components of the full-field ERGs had improved after chemotherapy, the amplitudes had still not reached normal limits (Figure 6). The recovery of the b-wave was greater than that of the a-wave in the bright flash ERG, and so the waveform was not of the "negative type" at this point.

Three years after the therapy, the patient had no evidence of systemic disease or recurrence of the PIOL. The ERGs were greatly improved but the amplitudes had still not reached the normal range.

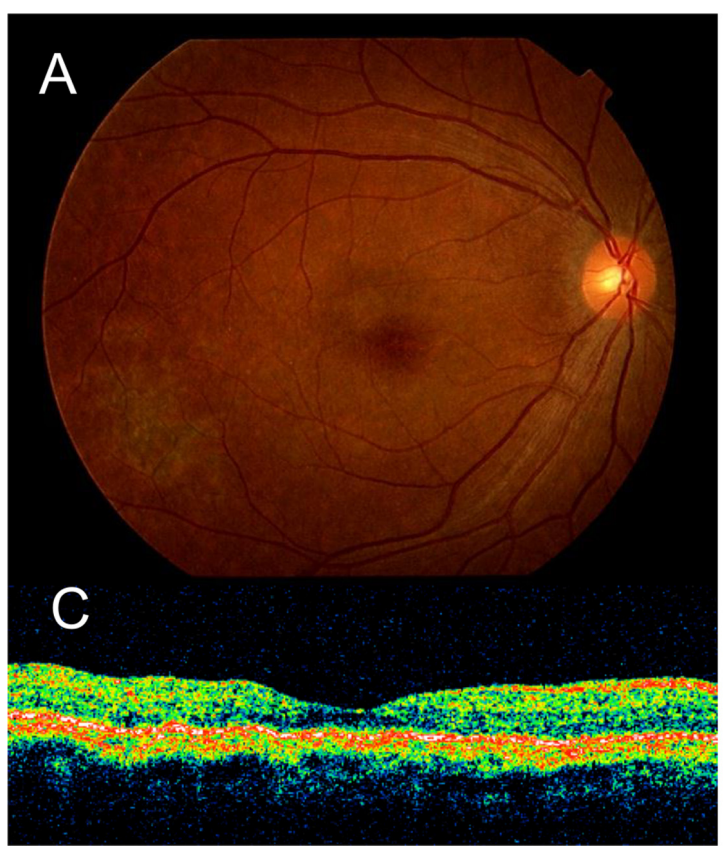

\section{Discussion}

We have followed a patient with a PIOL by electroretinography, who went into complete remission after chemotherapy. We found that the bright flash ERGs were the negative-type ERGs before chemotherapy. These ERG findings suggest that the inner retina was damaged more than the outer retina. As a differential diagnosis, we suspected endophthalmitis and uveitis, especially Beçhet's disease. However, from the history of intracranial malignant lymphoma, and the clinical signs, we diagnosed the patient as having a PIOL, even though the vitreous biopsy did not detect any malignant cells or a change

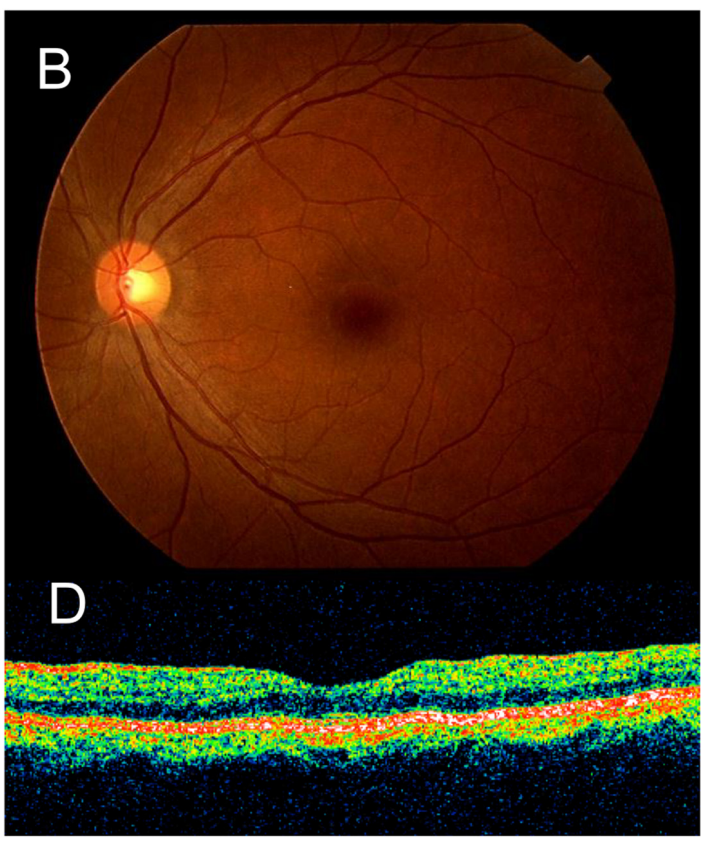

Figure 5 Fundus photography of the right $\mathbf{A}$ ) and the left eye $\mathbf{B}$ ) one month after chemotherapy. The ocular lesions have completely resolved leaving atrophic pigmentary changes. Optical coherence tomography of the macular area of the right $\mathbf{C}$ ) and the left eye $\mathbf{D}$ ) shows that the massive subretinal infiltration of the right eye has disappeared. 


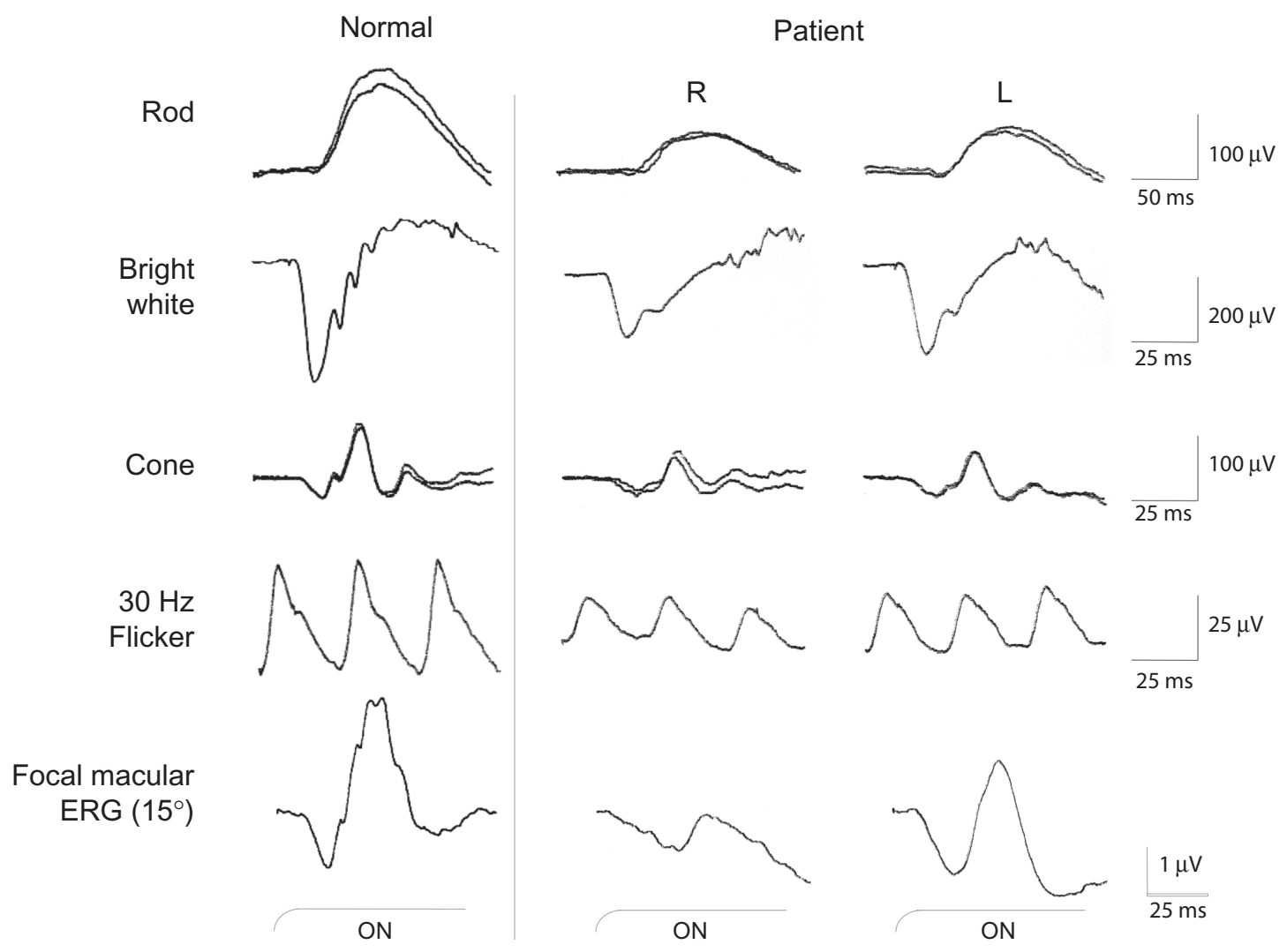

Figure 6 Full field ERGs (rod, bright white, cone and $30 \mathrm{~Hz}$ flicker) and focal macular ERG recorded one month after chemotherapy. The ERG amplitudes of all components have increased after therapy but still remained smaller than normal ERGS in both eyes.

in the IL-10/IL-6 ratio. It is known that the interpretation of vitreous specimens is sometimes difficult, and more than $40 \%$ of vitrectomy specimens may be nondiagnostic. ${ }^{8,3}$

After chemotherapy, the b-wave recovered more quickly than the a-wave. These results indicate that the inner retinal damage was transient, and may have resulted from secondary inflammation due to the presence of tumor cells. In the convalescent stage, when the ocular manifestations were resolved, both full-field and focal macular ERGs were still not fully recovered.

Earlier histopathological studies of PIOL's have mentioned an infiltration of malignant lymphoma cells into the subretinal space, which would be detected as whitish exudates, and could also lead to damage to the retina..$^{9,10}$ In our case, a marked prolongation of the focal macular ERG in the right eye may have been as a result of photoreceptor damage caused by just such subretinal infiltration.

\section{Conclusion}

In conclusion, we recorded ERGs from a patient whose ocular findings and history indicated that she had PIOL. The full-field ERGs were reduced in the right eye, showing a the negative-type ERG. After chemotherapy, there was a recovery in both the ocular signs and the ERGs. Although our conclusions are based on only one case, we recommend that ERGs be used to diagnose and follow patients with a PIOL.

\section{Acknowledgments/disclosures}

The authors report no conflicts of interest in this work, but acknowledge support from the Ministry of Education, Science, Sports and Culture, Japan - Grant number 18591913 (MK).

\section{References}

1. Tsai T, O’Brien JM. Masquerade syndromes: malignancies mimicking inflammation in the eye. Int Ophthalmol Clin. 2002;42(1):115-131.

2. Chan CC, Buggage RR, Nussenblatt RB. Intraocular lymphoma. Curr Opin Ophthalmol. 2002;13(6):411-418.

3. Coupland SE, Heimann H, Bechrakis NE. Primary intraocular lymphoma: a review of the clinical, histopathological and molecular biological features. Graefes Arch Clin Exp Ophthalmol. 2004;242(11):901-913.

4. Freeman LN, Schachat AP, Knox DL, Michels RG, Green WR. Clinical features, laboratory investigations, and survival in ocular reticulum cell sarcoma. Ophthalmology. 1987;94(12):1631-1639.

5. Marmor MF, Holder GE, Seeliger MW, Yamamoto S; International Society for Clinical Electrophysiology of Vision. Standard for clinical electroretinography (2004 update). Doc Ophthalmol. 2004;108(2): $107-114$. 
6. Miyake Y, Yagasaki K, Horiguchi M, Kawase Y, Kanda T. Congenital stationary night blindness with negative electroretinogram. A new classification. Arch Ophthalmol. 1986;104(7):1013-1020.

7. Miyake Y, Shiroyama N, Horiguchi M, Ota I. Asymmetry of focal ERG in human macular region. Invest Ophthalmol Vis Sci. 1989;30(8): $1743-1749$.

8. Coupland SE, Bechrakis NE, Anastassiou G, et al. Evaluation of vitrectomy specimens and chorioretinal biopsies in the diagnosis of primary intraocular lymphoma in patients with Masquerade syndrome. Graefes Arch Clin Exp Ophthalmol. 2003;241(10):860-870.
9. Chan CC, Wallace DJ. Intraocular lymphoma: update on diagnosis and management. Cancer Control. 2004;11(5):285-295.

10. Gill MK, Jampol LM. Variations in the presentation of primary intraocular lymphoma: case reports and a review. Surv Ophthalmol. 2001;45(6):463-471.
Clinical Ophthalmology

\section{Publish your work in this journal}

Clinical Ophthalmology is an international, peer-reviewed journal covering all subspecialties within ophthalmology. Key topics include: Optometry; Visual science; Pharmacology and drug therapy in eye diseases; Basic Sciences; Primary and Secondary eye care; Patient Safety and Quality of Care Improvements. This journal is indexed on

\section{Dovepress}

PubMed Central and CAS, and is the official journal of The Society of Clinical Ophthalmology (SCO). The manuscript management system is completely online and includes a very quick and fair peer-review system, which is all easy to use. Visit http://www.dovepress.com/ testimonials.php to read real quotes from published authors. 